\section{(2) OPEN ACCESS}

\title{
Interstitial lung disease is a risk factor for ischaemic heart disease and myocardial infarction
}

\author{
Lorna Elise Clarson 다, ${ }^{1}$ Ram Bajpai 다, ${ }^{1}$ Rebecca Whittle, ${ }^{1}$ John Belcher, ${ }^{1}$ \\ Alyshah Abdul Sultan, ${ }^{1}$ Chun Shing Kwok 이, ${ }^{2}$ Victoria Welsh, ${ }^{1}$ Mamas Mamas, ${ }^{2}$ \\ Christian D Mallen ${ }^{1}$
}

- Additional material is published online only. To view please visit the journal online (http://dx.doi.org/10.1136/ heartjil-2019-315511).

${ }^{1}$ School of Primary, Community and Social Care, Keele University, Keele, UK

${ }^{2}$ Cardiovascular Research Group Keele University, Stoke-onTrent, UK

Correspondence to Dr Lorna Elise Clarson, Keele University, Newcastle ST5 5BG, UK; I.clarson@keele.ac.uk

Received 3 June 2019 Revised 2 December 2019 Accepted 9 December 2019 Published Online First 29 February 2020
ABSTRACT

Despite many shared risk factors and pathophysiological pathways, the risk of ischaemic heart disease (IHD) and myocardial infarction (MI) in interstitial lung disease (ILD) remains poorly understood. This lack of data could be preventing patients who may benefit from screening for these cardiovascular diseases from receiving it.

Methods A population-based cohort study used electronic patient records from the Clinical Practice Research Datalink and linked Hospital Episode Statistics to identify 68572 patients (11688 ILD exposed (mean follow-up: 3.8 years); 56884 unexposed controls (mean follow-up: 4.0 years), with 349067 person-years of follow-up. ILD-exposed patients (pulmonary sarcoidosis (PS) or idiopathic pulmonary fibrosis (PF)) were matched (by age, sex, registered general practice and available follow-up time) to patients without ILD or IHD/MI. Rates of incident MI and IHD were estimated. HRs were modelled using multivariable Cox proportional hazards regression accounting for potential confounders.

Results ILD was independently associated with IHD (HR $1.85,95 \% \mathrm{Cl} 1.56$ to 2.18$)$ and $\mathrm{MI}(\mathrm{HR} 1.74,95 \% \mathrm{Cl}$ 1.44 to 2.11). In all disease categories, risk of both IHD and $\mathrm{MI}$ peaked between ages 60 and 69 years, except for the risk of $\mathrm{MI}$ in PS which was greatest $<50$ years. Men with PF were at greatest risk of IHD, while women with PF were at greatest risk of MI.

Conclusions ILD, particularly PF, is independently associated with $\mathrm{MI}$ and IHD after adjustment for established cardiovascular risk factors. Our results suggest clinicians should prioritise targeted assessment of cardiovascular risk in patients with ILD, particularly those aged 60-69years. Further research is needed to understand the impact of such an approach to risk management.

\section{BACKGROUND}

The interstitial lung diseases (ILDs) are a heterogenous group of diffuse parenchymal lung disorders characterised by fibrotic and/or inflammatory changes to the interstitial lung tissue. ${ }^{12}$ The most common of these conditions is idiopathic pulmonary fibrosis (PF), which affects between 14 and 63 per 100000 population in the USA, and between 1.25 and 23.4 per 100000 population in Europe. ${ }^{3}$ It is considered progressive and fatal; however, the course of the disease is variable with evidence suggesting comorbidity burden contributes significantly to risk of disease progression and excess mortality. ${ }^{4}$ Pulmonary sarcoidosis (PS) has traditionally been considered a more benign condition; however, an increasing trend in rates of mortality and hospitalisation of patients with sarcoidosis has been reported, with comorbid conditions thought to play an important role in this. ${ }^{6}$

Ischaemic heart disease (IHD) is prevalent among patients with ILD with rates of IHD among patients with interstitial pulmonary fibrosis (IPF) reported to be as high as $68 \% .{ }^{4}$ IHD is the second most common cause of death in patients with IPF, after IPF itself. ${ }^{7-9}$ The underlying pathogenesis of ILD and IHD share a number of pathophysiological mechanisms including oxidative stress, vascular endothelial injury and release of proinflammatory cytokines associated with a hypercoagulable state and formation of microthrombi. ${ }^{10}{ }^{11}$ Despite recognition of their coexistence, shared risk factors (RFs), the importance of burden of comorbidities in the disease course of ILD, and the availability of effective primary and secondary IHD prevention measures that could be considered to mitigate any increased risk of IHD in patients with ILD, research examining the risk of incident IHD in patients with ILD are few in number, small in terms of sample size and report lengths of follow-up potentially inadequate to detect the outcomes of interest. ${ }^{12-15}$

This study therefore aimed to investigate the risk of incident IHD and myocardial infarction (MI) in a large cohort of patients with ILD, compared with those without, using linked data from primary and secondary care.

\section{METHODS}

\section{Data source and study population}

The Clinical Practice Research Datalink (CPRD) is one of the largest databases of electronic primary care records in the world, covering approximately $7 \%$ of the UK population, and is representative of the broader UK population. Practices record patient demographics, consultations, hospitalisations, specialist referrals, prescriptions, test results, immunisations and diagnoses. Clinical information is entered using Read codes, a standard clinical terminology system used in general practice in the UK. Prescriptions are recorded using Multilex (or British National Formulary) codes. The quality of the data is regulated by the Medicines and Healthcare Products Regulatory Authority and only used when it has reached a certain standard of quality. 
High validity of diagnostic Read codes has been demonstrated within the CPRD. ${ }^{16}{ }^{17}$ CPRD data can now be linked with Hospital Episode Statistics (HES), which has details of all NHS inpatient care, hospital outpatient visits and emergency hospital attendance in England. Data from private patients treated in NHS hospitals and care delivered by treatment centres funded by the NHS are also included. Demographic data, along with discharge diagnoses and procedures, are recorded using the International Classification of Diseases version 10 and Operation and Procedure Coding Supplement version 4, respectively, and are linked to primary care data by a trusted third-party using NHS number, date of birth and sex. The CPRD-HES linked data covers more than $3 \%$ of the total English population and are representative of the general UK population. ${ }^{18}$

ILD exposure was defined as a recorded diagnosis of PS or PF (including idiopathic PF, cryptogenic fibrosing alveolitis and idiopathic fibrosing alveolitis) in their general practice electronic health record (EHR) between 1998 and 2017 using Read codes (available at https://www.keele.ac.uk/mrr). The date associated with the first-ever Read code for the conditions of interest was taken to be the date of diagnosis ('Index date'). Each incident case was matched to five controls without a diagnosis of either condition on age $( \pm 5$ years, sex, available follow-up time in CPRD ( \pm 3 years) and registered general practice. Unexposed patients were assigned the same index date as their matched ILD-exposed patient and for both, follow-up commenced from that index date. Those with a prior history of IHD (including coronary artery disease (CAD), angina and acute coronary syndrome) or MI, or less than 1 year of follow-up after the index date were excluded. Exposed and unexposed participants were required to be over age 18 years at diagnosis of ILD (or matched index date), have linkage to HES data to identify outcomes of interest, have no history of any of the outcomes of interest prior to the diagnosis of ILD and have 6-month up-to-standard data follow-up in CPRD.

The validity of diagnostic Read codes in CPRD for one of the rarer forms of inflammatory lung disease, cryptogenic fibrosing alveolitis, has been assessed by comparison with hospital documentation and was shown to be high with a positive predictive value of $95 \%{ }^{19}$

\section{Outcome definition}

The primary study outcomes were IHD (including CAD, angina and acute coronary syndrome) and MI identified using medical codes assigned by GPs, or in secondary care HES data (list of Read codes used to identify outcomes available at https://www. keele.ac.uk/mrr). Validity of general practice diagnoses has been reported to be high, with a positive predictive of $85.3 \%$ for circulatory diseases, ${ }^{17}$ and $82 \%-92 \%$ for acute MI. ${ }^{17}$

\section{Covariates}

Potential confounders considered were body mass index (BMI), smoking status and alcohol consumption (yes, no, ex or unknown), hypertension, hyperlipidaemia, diabetes, chronic kidney disease, prescription for cardiovascular drugs (defined as at least 30 days supply of an antihypertensive, antiplatelet or lipid-lowering medication), reported family history of cardiovascular disease and socioeconomic status defined based on the area in which the general practice at which the patient was registered is located (quintiles by rank of Indices of Multiple Deprivation). ${ }^{20}$ They were identified closest to the index date from both the primary care EHR and linked HES data.

\section{Statistical analysis}

Absolute rates (ARs) of IHD and MI per 10000 person-years (PY) and 95\% CIs were calculated for cases and controls. Cox proportional hazards regression was used to calculate HRs adjusted for the stated confounding factors. Those with missing BMI data were categorised into a separate 'missing' category and included in the analysis, as there is evidence that in this database, BMI data cannot be assumed to be missing completely at random, and therefore may not satisfy the 'missing at random' assumption required for multiple imputation. ${ }^{21}$ The proportional hazards assumption was tested using Schoenfeld residuals plot. All statistical analyses were performed using Stata V.15.1.This study was reviewed and approved by the CPRD's in-house Independent Scientific Advisory Committee (ISAC) reference number: 15_214RA

As we have a matched sample, this introduces a bias that must be accounted for in the analysis stage. Matched subjects will have correlation (greater similarity) in outcomes than two randomly selected subjects. This is because their baseline covariates are more similar, and baseline covariates are related to outcomes. We must therefore account for the lack of independence in outcomes that have been induced by matching. Hence, to account for the matched nature of the sample, we use a robust variance estimator that accounts for the clustering within matched sets. ${ }^{22}$

\section{Sensitivity analysis}

Two sensitivity analyses were performed. First, analyses were repeated removing patients with a coded diagnosis of cardiac sarcoidosis during the time period of observation in order to understand the impact of sarcoidosis affecting the cardiac system on our findings. Second, analyses were repeated introducing number of GP consultations during the period of observation as a variable to investigate the potential impact of surveillance bias.

\section{Patient and public involvement}

Patients were involved in prioritising the research question but, due to the nature of the study using a database of electronic healthcare records, they were not involved further in the design, conduct or reporting of this work.

\section{RESULTS}

\section{Basic characteristics}

Data were analysed for 11688 ILD-exposed participants, and 56884 unexposed matched controls, over a total of 349067 PY. Table 1 presents the characteristics of participants.

Patients with PS were younger, had a lower burden of cardiovascular RF and longer median duration of follow-up than either patients with PF or controls.

\section{Myocardial infarction}

The AR of MI was highest in patients with PF (AR 72.57 (95\% CI 62.95 to 83.65 ) per $10000 \mathrm{PY}$ ). Rates of MI were lower in those exposed to PS than unexposed patients (16.01 (95\% CI 12.17 to 21.07$)$ vs $32.26(95 \%$ CI 30.26 to 34.39$)$ per 10000 PY) (table 2).

Risk of MI compared with unexposed patients was similar between the ILD combined group patients and PF group (HR 1.53 (95\% CI 1.33 to 1.77 vs HR 1.58 (95\%CI 1.35 to 1.85$)$ ). The PS group was not at increased risk of MI. While for men, risks of MI were similar across each of the disease categories, women with PF were at greatest risk of MI and greater than their male counterparts. In ILD and PF risk of MI peaked between 


\begin{tabular}{|c|c|c|c|c|}
\hline & $\begin{array}{l}\text { ILD } \\
(n=11688)\end{array}$ & $\begin{array}{l}\text { PS } \\
(n=4568)\end{array}$ & $\begin{array}{l}P F \\
(n=7120)\end{array}$ & $\begin{array}{l}\text { Non-ILD controls } \\
(n=56884)\end{array}$ \\
\hline Male, n (\%) & $6291(53.8)$ & $2313(50.6)$ & $3978(55.8)$ & $31854(56.0)$ \\
\hline Median follow-up, years (IQR) & $3.8(1.8-7.3)$ & $6.1(2.8-10.5)$ & $3.0(1.5-5.4)$ & $4.0(2.0-7.4)$ \\
\hline Hypertension, $\mathrm{n}(\%)$ & $3577(30.6)$ & $752(16.5)$ & $2825(39.7)$ & $17030(29.9)$ \\
\hline Chronic kidney disease, n (\%) & $1173(10.0)$ & $179(3.9)$ & $994(14.0)$ & $3998(7.0)$ \\
\hline \multicolumn{5}{|l|}{$\operatorname{BMI}\left(\mathrm{kg} / \mathrm{m}^{2}\right)^{*}$} \\
\hline Normal $(\geq 18.5 \leq 24.9)$ & 3430 (29.4) & $1256(27.5)$ & $2174(30.5)$ & $16983(30.0)$ \\
\hline Underweight $(<18.5)$ & $289(2.5)$ & $63(1.4)$ & $226(3.2)$ & $1166(2.1)$ \\
\hline Overweight ( $\geq 25.0 \leq 29.9$ ) & $4081(34.9)$ & $1528(33.5)$ & $2553(35.9)$ & $17768(31.2)$ \\
\hline Yes & $8058(68.9)$ & $3221(70.5)$ & $4837(67.9)$ & $36533(64.2)$ \\
\hline Ex & $356(3.05)$ & $89(1.9)$ & $267(3.8)$ & $1395(2.5)$ \\
\hline Not recorded & $988(8.45)$ & $422(9.2)$ & $566(7.9)$ & $9672(17.0)$ \\
\hline \multicolumn{5}{|l|}{ Smoking history } \\
\hline No & $5867(50.2)$ & $3005(65.8)$ & $2862(40.2)$ & $27406(48.2)$ \\
\hline Yes & $1453(12.4)$ & $518(11.3)$ & $935(13.1)$ & $9406(16.5)$ \\
\hline Ex & $4188(35.8)$ & $986(21.6)$ & $3202(45.0)$ & $14579(25.6)$ \\
\hline Not recorded & $180(1.5)$ & $59(1.3)$ & $121(1.7)$ & $5493(9.7)$ \\
\hline Family history of CVD & $2410(20.6)$ & $920(20.1)$ & $1490(20.9)$ & $10545(18.5)$ \\
\hline \multicolumn{5}{|c|}{ Socioeconomic status (quintiles of Index of Multiple Deprivation) } \\
\hline Antiplatelet & $768(6.6)$ & $101(2.2)$ & $667(9.4)$ & $3160(5.6)$ \\
\hline Lipid lowering & $1071(9.2)$ & $235(5.1)$ & $836(11.7)$ & $4449(7.8)$ \\
\hline
\end{tabular}

*WHO Classification of Obesity According to BMI.

BMI, body mass index; CVD, cardiovascular disease; ILD, inflammatory lung disease; PF, pulmonary fibrosis; PS, pulmonary sarcoidosis.

ages 60 years and 69 years; however, in PS the youngest cohort of patients ( $<50$ years) were at greatest risk.

\section{Ischaemic heart disease}

The AR of IHD was also highest in patients with PF (102.61 (95\% CI 91.06 to 115.64$)$ per $10000 \mathrm{PY}$ ). Rates of IHD were also lower in those exposed to PS than unexposed patients (20.09 (95\% CI 15.73 to 25.67 ) vs 39.71 (95\% CI 37.49 to 42.07) per $10000 \mathrm{PY}$ ) (table 3).

ILD exposure was associated with a $59 \%$ increased risk of IHD compared with unexposed patients (HR 1.58, 95\% CI 1.37 to 1.83). Patients with PF were at greatest risk (HR 1.78, 95\% CI 1.51 to 2.09 ), while patients with PS were not at any increased risk of IHD.

Risk of IHD was greatest in men, while risk of MI was greatest in women (table 4; figure 1). Risk of both IHD and MI peaked in the 60-69 age group, with the exception of PS where risk of MI was greatest in patients $<50$ years (table 4 ; figure 2 ).

Results of the sensitivity analysis excluding patients with cardiac sarcoidosis did not reveal any material change in the findings (online supplementary table S1). The associations identified in the main analysis were strengthened in the sensitivity analysis adjusting for number of GP consultations (online supplementary table S2).

\section{CONCLUSION}

\section{Main findings}

Both men and women with ILD were at increased risk of IHD and MI compared with unexposed controls. PF patients have a 2-3 fold greater incidence of acute myocardial infarction (AMI) and IHD in younger age groups compared with PS and unexposed controls. Women with PF were at greatest risk of MI (approximately $80 \%$ excess risk), while men with PF were at greatest risk of IHD (also approximately $80 \%$ excess risk). Women with PS were not at any increased risk of IHD or MI. Men with ILD overall experienced a slightly higher risk of IHD and MI to that in women. In all disease categories, risk of both IHD and MI peaked between ages 60 years and 69 years, except for the risk of MI in PS which was greatest in the youngest age group ( $<50$ years). 
Table 2 Myocardial infarction incidence rates per 10000 person-year of follow-up by exposure status

\begin{tabular}{|c|c|c|c|c|c|c|c|c|c|c|c|c|}
\hline \multirow[b]{2}{*}{ Variables } & \multicolumn{3}{|c|}{ Unexposed } & \multicolumn{3}{|c|}{ ILD combined } & \multicolumn{3}{|l|}{ PS } & \multicolumn{3}{|l|}{ PF } \\
\hline & $\mathrm{N}$ & PY & Rate $^{*}(95 \% \mathrm{Cl})$ & $\mathrm{N}$ & PY & Rate $^{*}(95 \% \mathrm{Cl})$ & $\mathrm{N}$ & PY & Rate $^{*}(95 \% \mathrm{Cl})$ & $\mathrm{N}$ & PY & Rate $^{*}(95 \% \mathrm{Cl})$ \\
\hline Overall & 56884 & 291038 & $\begin{array}{l}32.26 \text { ( } 30.26 \text { to } \\
34.39)\end{array}$ & 11688 & 58029 & $\begin{array}{l}41.53 \text { (36.60 to } \\
47.12)\end{array}$ & 4568 & 31846 & $\begin{array}{l}16.01 \text { (12.17 to } \\
21.07)\end{array}$ & 7120 & 26183 & $\begin{array}{l}72.57 \text { (62.95 to } \\
83.65)\end{array}$ \\
\hline \multicolumn{13}{|l|}{ Sex } \\
\hline Male & 31854 & 151469 & $\begin{array}{l}40.27 \text { (37.20 to } \\
43.60)\end{array}$ & 6291 & 29195 & $\begin{array}{l}54.12 \text { (46.31 to } \\
63.25)\end{array}$ & 2313 & 15473 & $\begin{array}{l}20.68 \text { (14.63 to } \\
29.24)\end{array}$ & 3978 & 13722 & $\begin{array}{l}91.83 \text { (77.11 to } \\
109.34)\end{array}$ \\
\hline Female & 25030 & 139569 & $\begin{array}{l}23.57(21.16 \text { to } \\
26.26)\end{array}$ & 5397 & 28835 & $\begin{array}{l}28.78 \text { ( } 23.21 \text { to } \\
35.69)\end{array}$ & 2255 & 16373 & $\begin{array}{l}11.60 \text { (7.40 to } \\
18.19)\end{array}$ & 3142 & 12462 & $\begin{array}{l}51.36 \text { ( } 40.20 \text { to } \\
65.62)\end{array}$ \\
\hline \multicolumn{13}{|l|}{ Age (years) } \\
\hline$<50$ & 12272 & 94517 & $\begin{array}{l}4.97(3.74 \text { to } \\
6.62)\end{array}$ & 3075 & 22576 & $\begin{array}{l}10.63 \text { (7.13 to } \\
15.86)\end{array}$ & 2691 & 20207 & $\begin{array}{l}7.92 \text { (4.85 to } \\
12.92)\end{array}$ & 384 & 2369 & $\begin{array}{l}33.77(16.89 \text { to } \\
67.51)\end{array}$ \\
\hline $50-59$ & 7460 & 48505 & $\begin{array}{l}16.29(13.06 \text { to } \\
20.31)\end{array}$ & 1759 & 10830 & $\begin{array}{l}27.70(19.37 \text { to } \\
39.62)\end{array}$ & 1047 & 7091 & $\begin{array}{l}21.15(12.75 \text { to } \\
35.09)\end{array}$ & 712 & 3740 & $\begin{array}{l}40.11(24.18 \text { to } \\
66.53)\end{array}$ \\
\hline $60-69$ & 11321 & 57.911 & $\begin{array}{l}30.39(26.22 \text { to } \\
35.23)\end{array}$ & 2308 & 10643 & $\begin{array}{l}63.89 \text { (50.38 to } \\
81.04)\end{array}$ & 568 & 3376 & $\begin{array}{l}29.62(15.94 \text { to } \\
55.04)\end{array}$ & 1740 & 7267 & $\begin{array}{l}79.81(61.71 \text { to } \\
103.24)\end{array}$ \\
\hline $70-79$ & 15392 & 60347 & $\begin{array}{l}64.63 \text { (58.52 to } \\
71.37)\end{array}$ & 2759 & 9443 & $\begin{array}{l}80.48 \text { (64.27 to } \\
100.77)\end{array}$ & 210 & 1004 & $\begin{array}{l}89.60(46.62 \text { to } \\
172.21)\end{array}$ & 2549 & 8439 & $\begin{array}{l}79.39(62.49 \text { to } \\
100.87)\end{array}$ \\
\hline$>80$ & 10438 & 29757 & $\begin{array}{l}83.01 \text { (73.27 to } \\
94.03)\end{array}$ & 1786 & 4536 & $\begin{array}{l}94.79(70.30 \text { to } \\
127.81)\end{array}$ & 52 & 168 & $\begin{array}{l}59.67 \text { (8.41 to } \\
423.59)\end{array}$ & 1735 & 4369 & $\begin{array}{l}96.13(71.05 \text { to } \\
130.09)\end{array}$ \\
\hline
\end{tabular}

*Rate per 10000 person-years.

ILD, inflammatory lung disease; N, number; PF, pulmonary fibrosis; PY, person-years.

\section{Strengths of this study}

This study is the first to report the risk of MI in ILD and to report risks of IHD and MI by age and gender, identifying women with PF and patients $<50$ years with PS to be at greatest risk of MI. This study is also the first to adjust for important factors such as deprivation. The use of GP electronic medical records that are kept contemporaneously limits the risk of recall or observer bias and allows a long duration of patient follow-up. In addition, our study used linked secondary care data, which have been shown to ascertain outcomes more robustly. ${ }^{23}$

However, the findings of this study should be interpreted with caution due to the risk of misclassification resulting from the use of EHRs, although the validity of certain forms of inflammatory lung disease, including IPF diagnosis has previously been examined in such databases and was found to be high. ${ }^{20}$ The risk of surveillance bias should also be considered as it is possible that patients with ILD see their GP more frequently or are investigated in more detail than those in the general population that may lead to increased opportunities for detection of disease, although the findings of our sensitivity analysis would refute this. Finally, while we adjust for the presence of RFs such as hypertension, we do not adjust for their severity, and there may be a differential impact on risk across a range of blood pressure values.

\section{Interpretation in the context of other studies}

While other studies do not report risk of IHD by age and sex, our overall findings are in line with those of previously published studies reporting an increased risk of IHD in patients with ILD. ${ }^{912-1624-26}$ Two smaller studies that examined this association in an alternative database of UK primary care EHRs (The Health Improvement Network) reported an increased incidence of CAD or angina associated with IPF. After adjustment for age, sex and smoking status, Hubbard et al ${ }^{12}$ reported a rate ratio of incident CAD in 920 patients with IPF compared with 3593 controls of

Table 3 Ischaemic heart disease incidence rates per 10000 person-year (PY) of follow-up by exposure status

\begin{tabular}{|c|c|c|c|c|c|c|c|c|c|c|c|c|}
\hline \multirow[b]{2}{*}{ Variables } & \multicolumn{3}{|c|}{ Unexposed } & \multicolumn{4}{|c|}{ ILD combined } & \multicolumn{3}{|l|}{ PS } & \multicolumn{2}{|l|}{ PF } \\
\hline & $\mathrm{N}$ & PY & Rate* $(95 \% \mathrm{Cl})$ & $\mathrm{N}$ & PY & Rate $^{*}(95 \% \mathrm{Cl})$ & $\mathrm{N}$ & PY & Rate $^{*}(95 \% \mathrm{Cl})$ & $\mathrm{N}$ & PY & Rate* $(95 \% \mathrm{Cl})$ \\
\hline Overall & 56884 & 291098 & $\begin{array}{l}39.71 \text { (37.49 to } \\
42.07)\end{array}$ & 11688 & 58064 & $\begin{array}{l}57.35 \text { (51.51 to } \\
63.85)\end{array}$ & 4568 & 31849 & $\begin{array}{l}20.09 \text { (15.73 to } \\
25.67)\end{array}$ & 7120 & 26215 & $\begin{array}{l}102.61 \text { (91.06 to } \\
115.64)\end{array}$ \\
\hline \multicolumn{13}{|l|}{ Sex } \\
\hline Male & 31854 & 151493 & $\begin{array}{l}52.74(49.21 \text { to } \\
56.53)\end{array}$ & 6291 & 29209 & $\begin{array}{l}78.06 \text { ( } 68.56 \text { to } \\
88.88)\end{array}$ & 2313 & 15471 & $\begin{array}{l}20.68 \text { (14.63 to } \\
29.25)\end{array}$ & 3978 & 13737 & $\begin{array}{l}142.68(124.03 \text { to } \\
164.12)\end{array}$ \\
\hline Female & 25030 & 139605 & $\begin{array}{l}25.57(23.05 \text { to } \\
28.37)\end{array}$ & 5397 & 28855 & $\begin{array}{l}36.39 \text { (30.05 to } \\
44.06)\end{array}$ & 2255 & 16377 & $\begin{array}{l}19.54(13.82 \text { to } \\
27.67)\end{array}$ & 3142 & 12477 & $\begin{array}{l}58.51 \text { (46.51 to } \\
73.59)\end{array}$ \\
\hline \multicolumn{13}{|l|}{ Age (years) } \\
\hline$<50$ & 12272 & 94514 & $\begin{array}{l}6.35(4.93 \text { to } \\
8.18)\end{array}$ & 3075 & 22579 & $\begin{array}{l}9.74(6.42 \text { to } \\
14.80)\end{array}$ & 2691 & 20210 & $\begin{array}{l}7.42(4.47 \text { to } \\
12.31)\end{array}$ & 384 & 2369 & $\begin{array}{l}29.55 \text { (14.09 to } \\
61.98)\end{array}$ \\
\hline $50-59$ & 7460 & 48513 & $\begin{array}{l}24.94 \text { ( } 20.87 \text { to } \\
29.81)\end{array}$ & 1759 & 10841 & $\begin{array}{l}37.82(27.85 \text { to } \\
51.36)\end{array}$ & 1047 & 7098 & $\begin{array}{l}29.59(19.29 \text { to } \\
45.38)\end{array}$ & 712 & 3740 & $\begin{array}{l}53.43 \text { (34.47 to } \\
82.82)\end{array}$ \\
\hline $60-69$ & 11321 & 57927 & $\begin{array}{l}50.06 \text { (44.62 to } \\
56.17)\end{array}$ & 2308 & 10638 & $\begin{array}{l}98.70(81.52 \text { to } \\
119.51)\end{array}$ & 568 & 3367 & $\begin{array}{l}62.37 \text { ( } 40.67 \text { to } \\
95.66)\end{array}$ & 1740 & 7271 & $\begin{array}{l}115.52 \text { (93.28 to } \\
143.07)\end{array}$ \\
\hline $70-79$ & 15392 & 60369 & $\begin{array}{l}79.68 \text { (72.86 to } \\
87.12)\end{array}$ & 2759 & 9460 & $\begin{array}{l}130.03 \text { (108.96 to } \\
155.16)\end{array}$ & 210 & 1006 & $\begin{array}{l}59.61(26.78 \text { to } \\
132.69)\end{array}$ & 2549 & 8453 & $\begin{array}{l}138.41 \text { (115.47 to } \\
165.91)\end{array}$ \\
\hline$>80$ & 10438 & 29774 & $\begin{array}{l}68.52(59.73 \text { to } \\
78.59)\end{array}$ & 1786 & 4546 & $\begin{array}{l}92.39(68.28 \text { to } \\
125.02)\end{array}$ & 52 & 168 & $\begin{array}{l}59.56 \text { (8.39 to } \\
422.79\end{array}$ & 1735 & 4377 & $\begin{array}{l}93.65(68.96 \text { to } \\
127.19)\end{array}$ \\
\hline
\end{tabular}


Table 4 Risk of ischaemic heart disease (IHD) and myocardial infarction (MI) by exposure status

\begin{tabular}{|c|c|c|c|c|c|c|}
\hline & IHD & & & MI & & \\
\hline & Male & & Female & Male & Fem & \\
\hline & HR $\left(95^{\circ}\right.$ & & HR $(95 \% \mathrm{Cl})$ & HR $(95 \% \mathrm{Cl})$ & $\mathrm{HR}$ & $6 \mathrm{Cl})$ \\
\hline ILD & $1.66(1$. & 1.98) & 1.36 (1.03 to 1.79$)$ & 1.55 (1.31 to 1 & 1.49 & 6 to 1.91$)$ \\
\hline PS & $1.13(0.7$ & 1.63) & 0.88 (0.55 to 1.41$)$ & 1.55 (1.13 to 2 & 0.89 & 6 to 1.42$)$ \\
\hline PF & $1.81(1.5$ & 2.18) & 1.67 (1.22 to 2.30$)$ & 1.51 (1.24 to 1 & 1.80 & 6 to 2.38$)$ \\
\hline & IHD (HR $(95 \% \mathrm{Cl}))$ & & & MI (HR $(95 \% \mathrm{Cl}))$ & & \\
\hline Age (years) & ILD & PS & PF & ILD & PS & $\mathrm{PF}$ \\
\hline$<50$ & 1.61 (0.92 to 2.81$)$ & 1.49 (0.83 to 2.66$)$ & 1.89 (0.56 to 6.33$)$ & 1.72 (1.04 to 2.83$)$ & 1.83 (1.10 to 3.05$)$ & 0.87 (0.21 to 3.66$)$ \\
\hline 50-59 & 1.16 (0.73 to 1.82$)$ & 0.75 (0.41 to 1.36$)$ & 2.05 (1.13 to 3.72 ) & 1.48 (0.95 to 2.29$)$ & 1.35 (0.80 to 2.28$)$ & 1.47 (0.78 to 2.74$)$ \\
\hline 60-69 & 1.92 (1.46 to 2.52 ) & $0.88(0.51$ to 1.53$)$ & 2.40 (1.79 to 3.22 ) & 1.79 (1.35 to 2.38$)$ & 0.62 (0.32 to 1.22$)$ & 2.35 (1.74 to 3.16$)$ \\
\hline $70-79$ & 1.71 (1.35 to 2.17$)$ & 0.81 (0.36 to 1.81$)$ & 1.84 (1.45 to 2.35$)$ & 1.77 (1.40 to 2.23 ) & 1.35 (0.72 to 2.54$)$ & 1.79 (1.40 to 2.29$)$ \\
\hline$>80$ & $1.08(0.73$ to 1.61$)$ & $0.82(0.11$ to 6.18$)$ & 1.10 (0.73 to 1.64$)$ & 0.91 (0.64 to 1.28$)$ & $1.30(0.38$ to 4.39$)$ & 0.89 (0.62 to 1.27$)$ \\
\hline
\end{tabular}

All analyses adjusted for CKD, HTN, DM, HLD, BMI, exposure to smoking and alcohol, IMD, family history of cardiovascular disease and exposure to antihypertensive, antiplatelet and lipid-lowering drugs.

BMI, body mass index; CKD, chronic kidney disease; DM, diabetes mellitus; HLD, hyperlipidaemia; HR, adjusted HR; HTN, hypertension; ILD, inflammatory lung disease; IMD, Index of Multiple Deprivation; PF, pulmonary fibrosis; PS, pulmonary sarcoidosis.

3.39 (95\% CI 2.02 to 4.87 ), which is slightly higher than the rate ratio in this study of 2.39 (95\% CI 2.11 to 2.70 ) but may reflect the wider range of cardiovascular RFs adjusted for in this study. Dalleywater et al reported a rate ratio of IHD $2.32(95 \%$ CI 1.85 to 2.93) in 3211 participants with IPF compared with 12307 controls, after adjustment for hypertension, hypercholesterolaemia, diabetes, smoking status and BMI, similar to the findings of this study. ${ }^{15}$ A further study of 406 Korean patients with IPF reported that the incidence of CAD was higher $(6.8 \%)$ compared with controls $(2.8 \%)$ with a relative risk of $1.92(95 \%$

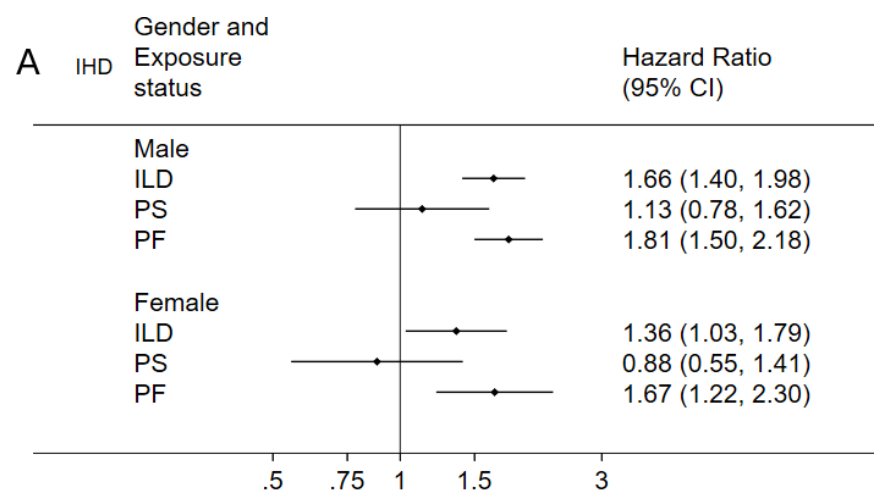

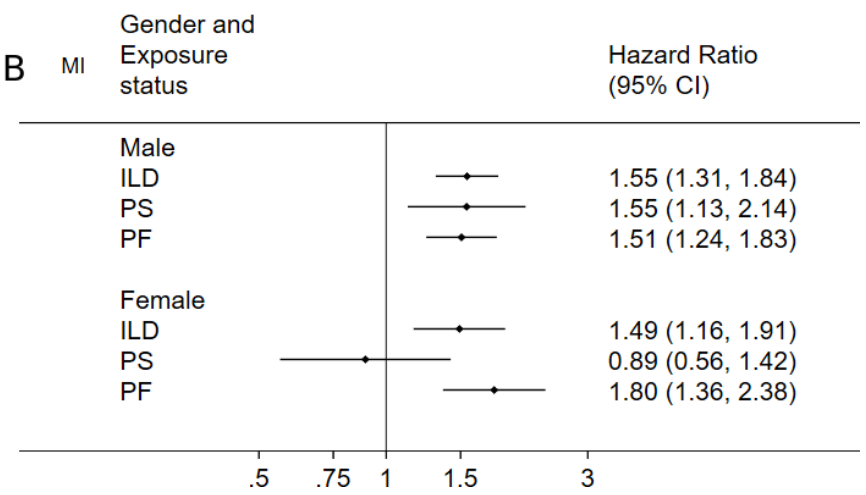

Figure 1 Risk of IHD (A) and myocardial infarction (B) by gender and exposure status. IHD, ischaemic heart disease; ILD, interstitial lung disease; PF, pulmonary fibrosis; PS, pulmonary sarcoidosis.
CI 1.08 to 3.43 ), also similar in magnitude to our findings. ${ }^{16}$ Increased prevalence of angiographically determined CAD in patients with PF has also been reported, with an OR of 2.18 for the presence of angiographic CAD in patients with fibrotic versus non-fibrotic lung disease. ${ }^{24}$ Significantly more CAD was reported in 49 patients with lung fibrosis (28.6\%) compared with 51 with emphysema $(9.8 \%, p=0.02$ for difference) despite there being significantly more smokers in the emphysema group (98\% vs 31\%), ${ }^{25}$ and prevalence of CAD was higher in pretransplant IPF patients $(65.8 \%)$ than pretransplant chronic obstructive pulmonary disease patients $(46.1 \%, \mathrm{p}=0.03$ for difference) independent of CAD RFs. IPF patients with severe CAD also had worse outcomes than controls with a similar disease burden in this study. ${ }^{26}$

In common with other studies, there was an increased prevalence of established cardiovascular RFs in our ILD and PF cohorts, ${ }^{15}$ and while we adjusted for the presence of these RFs, we could not adjust for their severity. It is therefore possible that this association reflects either less well controlled cardiovascular RFs in patients with ILD/PF or the presence of unmeasured novel cardiovascular RFs.
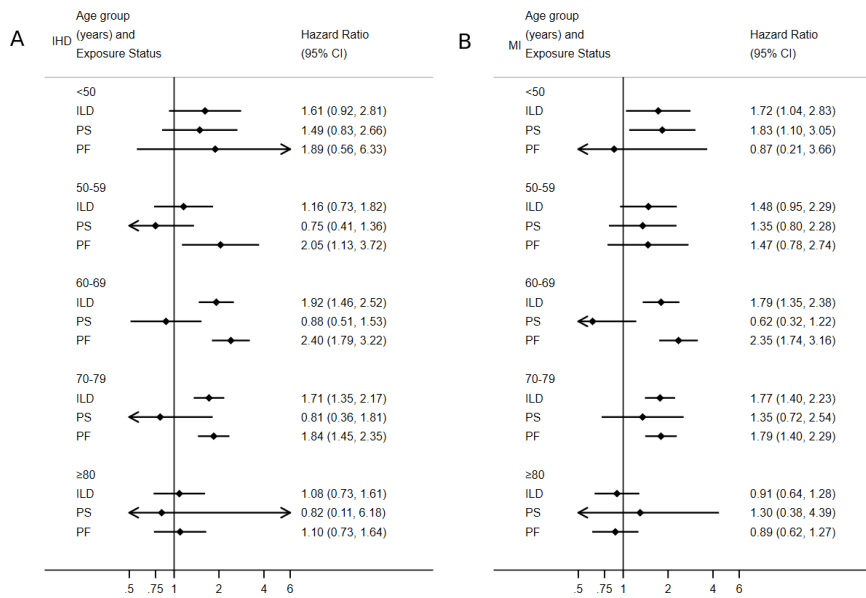

Figure 2 Risk of IHD (A) and myocardial infarction (B) by age and exposure status. IHD, ischaemic heart disease; ILD, interstitial lung disease; PF, pulmonary fibrosis; PS, pulmonary sarcoidosis. 
It has also been suggested that the presence of a serious lung disease might distract medical attention from routine cardiovascular care and that either primary and secondary prevention interventions are neglected, or symptoms that might be suggestive of cardiovascular disease are falsely attributed to the known respiratory condition. ${ }^{12}$

Several pathophysiological mechanisms may account for the association between ILD and CAD. Autoantibodies such as antinuclear antibody and antiendothelial cell antibodies are known to circulate in higher concentrations in patients with IPF than the general population, and deposition of such autoantibodies in the endothelial cells of lung tissue may stimulate the release of proinflammatory cytokines and monocyte recruitment and activation. ${ }^{10}$ In addition, oxidative stress may play a role. ${ }^{11}$ This triggering of the inflammatory cascade has been hypothesised to cause endothelial injury or necrosis and is also associated with a hypercoagulable state, the formation of microthrombi and the activation of fibroblasts in common with mechanisms that have been implicated in IHD and MI. ${ }^{10} 2728$ Patients with IPF have been reported to have an increased tendency to thromboembolic disease and to have higher factor VIII levels than the general population, with elevated factor VIII levels also associated with an increased risk of CAD. ${ }^{29}$

In common with other systemic inflammatory disorders, this study demonstrates that the presence of PF provides independent information to the incident risk of MI/IHD on top of classical cardiovascular RFs. This has led to both rheumatoid arthritis

Key messages

What is already known on this subject?

- In common with other inflammatory diseases, such as rheumatoid arthritis (RA) and systemic lupus erythematosus (SLE), interstitial lung disease (ILD) and ischaemic heart disease (IHD) share a number of pathophysiological mechanisms and common risk factors. However, unlike these other diseases, the risk of IHD in patients with ILD is poorly understood. This lack of data could be preventing patients with ILD who may benefit from screening for these cardiovascular diseases from receiving it, as patients with RA and SLE have.

\section{What might this study add?}

- This study is the first to report the risk of myocardial infarction (MI) in ILD and to report risks of IHD and MI by age and gender, identifying women with pulmonary fibrosis (PF) and patients $<50$ years with pulmonary sarcoidosis to be at greatest risk of MI. This study is also the first to adjust for important factors such as deprivation.

- This study demonstrates that the presence of PF provides independent information to the incident risk of MI/IHD on top of classical cardiovascular RFs.

\section{How might this impact on clinical practice?}

- The increase in risk of AMI/IHD associated with PF/ILD in this study exceeds that attributed to RA within the preferred model of newest iteration of the QRISK tool and in younger age groups also exceeds that attributed to SLE. It could therefore be argued that in common with recommendations for RA/SLE, clinicians should screen patients with ILD for IHD, and furthermore, ILD should be considered in future iterations of cardiovascular risk stratification tools.
(RA) and systemic lupus erythematosus (SLE) being included in cardiovascular risk stratification tools. ${ }^{30}$ The increase in risk of AMI/IHD associated with PF/ILD in this study exceeds that attributed to RA within the preferred model of newest iteration of the QRISK tool (HR men 1.23 (95\% CI 1.19 to 1.28); women 1.24 (95\% CI 1.20 to 1.27$)$ ) and in younger age groups also exceeds that attributed to SLE (HR men 1.55 (95\% CI 1.15 to 2.10); women 2.14 (95\% CI 1.78 to 2.56)). ${ }^{30}$ It could therefore be argued that ILD should be considered in future iterations of cardiovascular risk stratification tools.

\section{Conclusion}

Our results enable clinicians to identify which cohorts of ILD patients are most at risk of IHD and MI and target their assessment of cardiovascular risk appropriately. Our results also suggest that a more aggressive approach to primary prevention may be warranted in certain groups of ILD patients, for example, those $<50$ years old with PS. Further research is needed to understand the impact of such a stratified approach to risk management.

Twitter Ram Bajpai @RamBajpai16 and Chun Shing Kwok @DrShingKwok

Contributors All authors were involved in the inception and design of the study. $L C, R B$ and JB were responsible for analysis of the data. All authors were involved in the interpretation of the data and the preparation of the manuscript.

Funding This work was supported by the National Institute for Health Research School for Primary Care Research Grant Number: 255. Clarson and Welsh are funded by NIHR Academic Clinical Lectureships. Mallen is funded by the NIHR Collaborations for Leadership in Applied Health Research and Care West Midlands, the NIHR School for Primary Care Research and a NIHR Research Professorship in General Practice, which also supports Bajpai (NIHR-RP-2014-04-026). The views expressed are those of the author(s) and not necessarily those of the National Health Service, the NIHR or the Department of Health and Social Care. The funder was not involved in the study design; in the collection, analysis and interpretation of data; in the writing of the report; or in the decision to submit the article for publication.

\section{Competing interests None declared.}

Patient and public involvement Patients and/or the public were not involved in the design, or conduct, or reporting, or dissemination plans of this research.

Patient consent for publication Not required.

Provenance and peer review Not commissioned; externally peer reviewed.

Data availability statement This study is based in part on data from the Clinical Practice Research Datalink obtained under licence from the UK Medicines and Healthcare products Regulatory Agency. The data is provided by patients and collected by the NHS as part of their care and support. The interpretation and conclusions contained in this study are those of the author/s alone. HES data Copyright (C) (2019), re-used with the permission of The Health \& Social Care Information Centre. All rights reserved. The data used in this study can only be used for the purposes set out in the submitted and approved ISAC protocol. No data can, therefore, be archived by the research team. Any future research would require a new application to CPRD with data obtained directly from CPRD subject to their policies for scientific, data governance, and financial approvals (see www.cprd.com).

Open access This is an open access article distributed in accordance with the Creative Commons Attribution 4.0 Unported (CC BY 4.0) license, which permits others to copy, redistribute, remix, transform and build upon this work for any purpose, provided the original work is properly cited, a link to the licence is given, and indication of whether changes were made. See: https://creativecommons.org/ licenses/by/4.0/.

\section{ORCID iDs}

Lorna Elise Clarson http://orcid.org/0000-0003-0828-9649

Ram Bajpai http://orcid.org/0000-0002-1227-2703

Chun Shing Kwok http://orcid.org/0000-0001-7047-1586

\section{REFERENCES}

1 Antoniou KM, Hansell DM, Rubens MB, et al. Idiopathic pulmonary fibrosis: outcome in relation to smoking status. Am J Respir Crit Care Med 2008;177:190-4.

2 Bagnato G, Harari S. Cellular interactions in the pathogenesis of interstitial lung diseases. Eur Respir Rev 2015;24:102-14.

3 Nalysnyk L, Cid-Ruzafa J, Rotella P, et al. Incidence and prevalence of idiopathic pulmonary fibrosis: review of the literature. Eur Respir Rev 2012;21:355-61. 
4 Raghu G, Amatto VC, Behr J, et al. Comorbidities in idiopathic pulmonary fibrosis patients: a systematic literature review. Eur Respir J 2015;46:1113-30.

5 Behr J, Kreuter M, Hoeper MM, et al. Management of patients with idiopathic pulmonary fibrosis in clinical practice: the INSIGHTS-IPF registry. Eur Respir J 2015;46:186-96.

6 Gerke AK. Morbidity and mortality in sarcoidosis. Curr Opin Pulm Med 2014;20:472-8.

7 Hutchinson J, Fogarty A, Hubbard R, et al. Global incidence and mortality of idiopathic pulmonary fibrosis: a systematic review. Eur Respir J 2015;46:795-806.

8 Schuett KA, Lehrke M, Marx N, et al. High-Risk cardiovascular patients: clinical features, comorbidities, and Interconnecting mechanisms. Front Immunol 2015;6:591.

9 Panos RJ, Mortenson RL, Niccoli SA, et al. Clinical deterioration in patients with idiopathic pulmonary fibrosis: causes and assessment. Am J Med 1990;88:396-404.

10 Bringardner BD, Baran CP, Eubank TD, et al. The role of inflammation in the pathogenesis of idiopathic pulmonary fibrosis. Antioxid Redox Signal 2008; 10:287-302.

11 Bargagli E, Olivieri C, Bennett $D$, et al. Oxidative stress in the pathogenesis of diffuse lung diseases: a review. Respir Med 2009;103:1245-56.

12 Hubbard RB, Smith C, Le Jeune I, et al. The association between idiopathic pulmonary fibrosis and vascular disease: a population-based study. Am J Respir Crit Care Med 2008; 178:1257-61.

13 Ponnuswamy A, Manikandan R, Sabetpour A, et al. Association between ischaemic heart disease and interstitial lung disease: a case-control study. Respir Med 2009;103:503-7.

14 Dalleywater W, Powell HA, Hubbard RB, et al. Risk factors for cardiovascular disease in people with idiopathic pulmonary fibrosis: a population-based study. Chest 2015;147:150-6.

15 Kim W-Y, Mok Y, Kim GW, et al. Association between idiopathic pulmonary fibrosis and coronary artery disease: a case-control study and cohort analysis. Sarcoidosis Vasc Diffuse Lung Dis 2015;31:289-96.

16 Herrett E, Thomas SL, Schoonen WM, et al. Validation and validity of diagnoses in the general practice research database: a systematic review. Br J Clin Pharmacol 2010;69:4-14.
17 Khan NF, Harrison SE, Rose PW. Validity of diagnostic coding within the general practice research database: a systematic review. Br J Gen Pract 2010;60:e128-36.

18 Herrett E, Gallagher AM, Bhaskaran K, et al. Data resource profile: clinical practice research Datalink (CPRD). Int J Epidemiol 2015;44:827-36.

19 Hubbard R, Venn A, Lewis S, et al. Lung cancer and cryptogenic fibrosing alveolitis. A population-based cohort study. Am J Respir Crit Care Med 2000;161:5-8.

20 Jordan H, Roderick P, Martin D. The index of multiple deprivation 2000 and accessibility effects on health. J Epidemiol Community Health 2004;58:250-7.

21 Bhaskaran K, Forbes HJ, Douglas I, et al. Representativeness and optimal use of body mass index (BMI) in the UK clinical practice research Datalink (CPRD). BMJ Open 2013;3:e003389.

22 Lin DY, Wei LJ. The robust inference for the COX proportional hazards model. J Am Stat Assoc 1989;84:1074-8.

23 Herrett $E$, Shah AD, Boggon R, et al. Completeness and diagnostic validity of recording acute myocardial infarction events in primary care, hospital care, disease registry, and national mortality records: cohort study. BMJ 2013;346:\{2350.

24 Kizer JR, Zisman DA, Blumenthal NP, et al. Association between pulmonary fibrosis and coronary artery disease. Arch Intern Med 2004;164:551-6.

25 Izbicki G, Ben-Dor I, Shitrit D, et al. The prevalence of coronary artery disease in end-stage pulmonary disease: is pulmonary fibrosis a risk factor? Respir Med 2009;103:1346-9.

26 Nathan SD, Basavaraj A, Reichner C, et al. Prevalence and impact of coronary artery disease in idiopathic pulmonary fibrosis. Respir Med 2010;104:1035-41.

27 Libby P. Inflammation in atherosclerosis. Nature 2002;420:868-74.

28 Navaratnam V, Fogarty AW, McKeever T, et al. Presence of a prothrombotic state in people with idiopathic pulmonary fibrosis: a population-based case-control study. Thorax 2014;69:207-15.

29 Meade TW, Cooper JA, Stirling Y, et al. Factor VIII, ABO blood group and the incidence of ischaemic heart disease. Br J Haematol 1994;88:601-7.

30 Hippisley-Cox J, Coupland C, Brindle P. Development and validation of QRISK3 risk prediction algorithms to estimate future risk of cardiovascular disease: prospective cohort study. BMJ 2017;357:j2099. 